\title{
On Similarities and Differences of Invasive and Non-Invasive Electrical Brain Signals in Brain-Computer Interfacing
}

\author{
David Steyrl, Reinmar J. Kobler, Gernot R. Müller-Putz \\ Institute of Neural Engineering, Laboratory of Brain-Computer Interfaces, Graz University of Technology, \\ Graz, Austria \\ Email: gernot.mueller@tugraz.at
}

Received 12 May 2016; accepted 26 June 2016; published 30 June 2016

Copyright $(2016$ by authors and Scientific Research Publishing Inc.

This work is licensed under the Creative Commons Attribution International License (CC BY). http://creativecommons.org/licenses/by/4.0/

(c) (1) Open Access

\begin{abstract}
We perceive that some Brain-Computer Interface (BCI) researchers believe in totally different origins of invasive and non-invasive electrical BCI signals. Based on available literature we argue, however, that although invasive and non-invasive BCI signals are different, the underlying origin of electrical BCIs signals is the same.
\end{abstract}

\section{Keywords}

Brain-Computer Interfaces, Electrical Brain Signals, Invasive Signals, Non-Invasive Signals, Comparison

\section{Introduction}

A Brain-Computer Interface (BCI) enables people to control computers and consequently devices directly via their thoughts. A BCI measures specific thought related brain activity and translates it into commands for communication and control [1] [2]. People affected by impaired motor functions, due to neurodegenerative diseases or traumata, can particularly benefit from a BCI [3] [4]. Today, however, people without disorders also benefit from a BCI. For example, a BCI can be used to assess, track and process actual mental states [5] [6].

BCIs can be divided according to their invasiveness into invasive and non-invasive BCIs, i.e., whether sensors used to measure brain activity are penetrating the skin or not. Over the last years particularly one type of invasive BCIs repeatedly gained attention in media and in the scientific community: invasive BCIs that used small grids of electrodes implanted directly in the motor cortex and related areas. In this context, motor imagery is used as an intuitive and natural strategy to elicit brain activity changes and subsequently to control movements 
of a robotic arm. Starting from the research with monkeys, case studies are available nowadays, demonstrating that the concept is also valid in humans [7]-[9]. In a setting with a robotic arm, a user was able to control up to ten different possible movement directions in real time [10]. We refer to this specific type of invasive BCIs when we use the term invasive BCI throughout this work. Despite the fact that the results of the case study show a high level of control, there is a critical issue with invasive BCIs: in a recent study, published by Blabe et al. (2015), 40\% of the respondents would not consider a neurosurgery at all, even if very good results could be expected [11]. In another study, conducted by Collinger et al. (2013), non-invasiveness was also rated as a high priority [12].

The vast majority of non-invasive BCIs are realized with electroencephalogram (EEG) measurements of brain activity [13]. Two concepts in non-invasive EEG based BCIs are common: 1) evoked response based BCIs and 2) induced response based BCIs [14]. Evoked response based BCIs have already proven to work satisfactorily for communication purposes, even in home environments and on a daily basis over years [15]-[18]. Evoked response based BCIs rely in their concept on external stimuli and generate control signals by shifting attention between stimuli. This artificial type of control can be irritating or tiring for users. A more intuitive control method is used in induced response based BCIs. These rely on changes in the EEG due to self-induced brain tasks such as motor imagery [19] [20]. This is the non-invasive counterpart of the above mentioned invasive BCI. We have this second concept in mind when we use the term non-invasive BCIs in this work. Using non-invasive BCIs to control neuroprostheses like a robotic arm is, however, a relatively challenging task. A review on that topic is presented in Rupp et al. (2015) [4]. Generally speaking, non-invasive motor imagery BCIs offer lower information transfer rates from brain to computer and consequently less possible movement directions or responsiveness.

Although invasive and non-invasive BCIs are based on electrical brain signals, we repeatedly perceived a controversy on the origin, or the underlying source of those signals. We perceived that some BCI researchers believe totally different origins of invasive and non-invasive BCIs signals. We think that the perceived controversy has several causes: 1) different research communities with a lack in exchange of information and different naming conventions, 2) differences in signal processing and system handling make them "feel” different, 3) differences in media coverage change the perception of the BCI systems. Based on available literature, our view is, however, that although invasive and non-invasive BCI signals are different, they rely on the same underlying source. To underpin our view, we provide a condensed and focused summary of available information on origins, similarities and differences of invasive and non-invasive electrical brain signals in the following sections, followed by a comparative discussion.

\section{Origin of Invasive BCI Signals}

Brain tissue is electrically active. Ionic currents cause local extracellular potential changes whose superposition is termed local field potential (LFP) [21]. The LFP has a broad frequency spectrum starting from DC up to several hundred hertz. Action potentials (AP) and synaptic transmissions are regarded as main sources of the LFP [22]. A plausible assumption is that APs are the source of high frequency components $(>500 \mathrm{~Hz})$ and synaptic transmissions the source of low frequency components [21]. The contribution of single sources to the LFP decays with the square of the distance if ohmic impedance and electric dipole sources are assumed [23]. The type of impedance of brain tissue is controversial. Typically brain tissue is assumed to have high pass properties, but there are also indications for low pass properties [24] [25].

However, a work of Logothetis et al. (2007) showed that pure ohmic impedance is a sufficient assumption for low frequencies $(<1000 \mathrm{~Hz}$ ) [26]. A recent modeling of the LFP conducted by Linden et al. (2011) brought up that for uncorrelated activity, a reach of $200 \mu \mathrm{m}$ is a realistic assumption. For correlated activity it is more difficult, but they concluded that “...the LFP recorded by an electrode is dominated by populations with substantial synaptic processes in the recording layer.” [27]. Hence, nearby sources contribute most to the LFP and distant sources contributions are subject to strong attenuation.

Invasive BCIs measure the LFP directly with tissue penetrating electrodes, typically with more than one electrode. Often a rectangular grid of about 100 microelectrodes referred to as microarray is applied. A common microelectrode length is $1.5 \mathrm{~mm}$ and was used in the invasive BCI experiments mentioned above [7]-[10]. The (motor) cortex is histologically divided into 6 layers and is about 3 to $4 \mathrm{~mm}$ thick [28]. The motor cortex is agranular, hence layer 4 is very thin. However, layer 3 compensates for layer 4 and is thicker [28]. Keeping in 
mind the spatial reach of the LFP and the electrode length, one can assume that the measured LFP is primarily generated in layers 1 to 4 (see Figure 1 right hand panel). Efferent fibers in layers 1 to 4 mainly connect to other cortical regions of the same and opposite hemisphere [28]. Afferent fibers in layers 1 to 4 mainly originate from other cortical regions, thalamus and brainstem [28]. This implies that primary sources of the measured LFP are internal processing inputs from the thalamus and brainstem, but not outputs to the motor system, as they start in layers 5 and 6 .

The numbers of APs per time unit-also termed firing rate-of neural populations in specific motor cortex areas are tightly correlated with movement directions and imagined/attempted ones [29] [30].

In summary, non-invasive BCIs directly measure the LFP in layers 1 to 4 of specific motor cortex areas and extract high frequency components to estimate AP firing rates. These firing rates are subsequently used to decode imagined/attempted movement directions.

\section{Origin of Non-Invasive BCI Signals}

EEG is by far the most common method for measuring electrical brain activity non-invasively. It measures potentials at the scalp using electrodes with a diameter of approximately $2 \mathrm{~mm}$ to $1 \mathrm{~cm}$. These scalp potentials are a modified version of the LFP in the brain [21]. The modification has at least two causes: 1) as mentioned above, the electric field decays with the square of the distance. Therefore, the LFP is subject to substantial attenuation until it reaches electrodes at the scalp; 2) Volume conductance of the heads tissues (brain, cerebral fluid, skull, scalp) cause spatial smoothing over an area of about $10 \mathrm{~cm}^{2}$ [21].

Due to the attenuation and smoothing, only synchronous brain activity, meaning brain activity that sum up over brain areas, can be measured at scalp level. Rhythms that occur synchronously are typical for lower frequency ranges of the LFP. One prominent example at motor cortex is the mu rhythm at $8-13 \mathrm{~Hz}$ [19]. The low

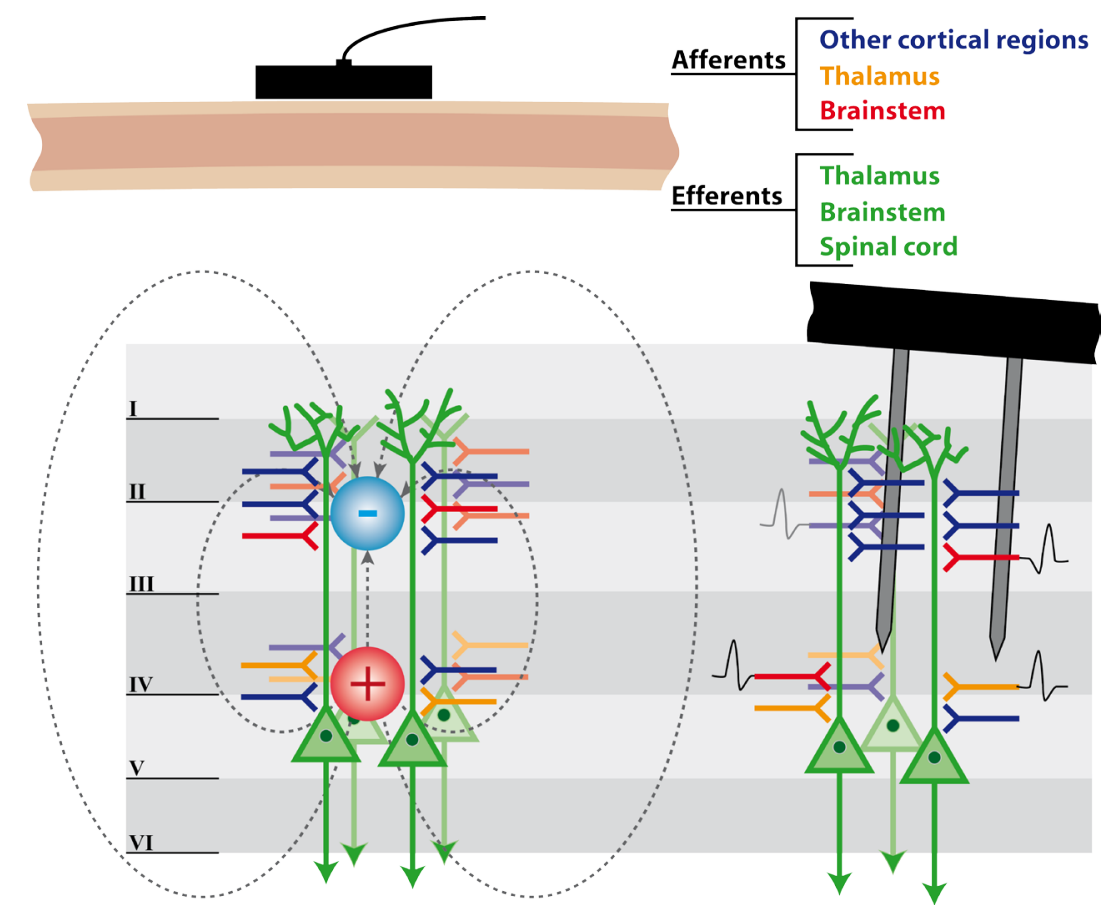

Figure 1. Sketch of BCI signal sources. I - VI mark the cortical layers. Cortical layer 5 and 6 pyramidal cells are highlighted in green. Their apical and basal synapses are color coded. The spatial and temporal dendritic integration of synaptic transmission leads to formation of dipoles (left side). If millions of neurons receive synchronous basal or apical synaptic transmissions, the resulting electrical field propagates over large distances and is even detectable at the scalp where it is called EEG. In contrast, implanted electrode arrays are capable of measuring APs due to their close vicinity to neurons (right side). Therefore, (presynaptic) action potentials can be recorded and decoded from LFP by signal processing. 
frequency components of LFP are mainly caused by correlated synaptic transmissions that form neural dipoles in parallel pyramid cells (see Figure 1 left hand panel) [21] [22]. Since only afferent APs cause synaptic transmissions, it can be assumed that the major contribution to LFP at lower frequencies comes from these afferent APs of cortex layers 1 to 4 (see Figure 1 left hand panel).

APs cause synaptic transmissions, but information coded in AP spike trains is not one-to-one equivalent to information in low frequency components of the LFP [22]. In fact, the connection from APs via synaptic transmissions to the LFP is not entirely understood yet. Ionic transmembrane currents can be well described by models, however influences like feedback of the LFP to surrounding cell activity, but also random effects, like synaptic transport failure limit our understanding [22] [31] [32].

In summary, APs of afferent fibers in the cortex can cause synaptic transmissions. Correlated synaptic transmissions form parallel neural dipoles which contribute most to synchronous low frequency components of the LFP and therefore also to the scalp EEG.

\section{Discussion}

Similarities: when comparing invasive and non-invasive BCI signals, one can find that the underlying sources are equal. At the very base, both signals' origins are AP spiking rates of cortical layers 1 to 4 . Hence, they both use internal processing inputs from thalamus and brainstem rather than outputs to the motor system.

Differences: invasive BCIs measure the LFP directly, whereas non-invasive BCIs only have access to a modified LFP. Known modifications are transformation from spiking to oscillatory activity, attenuation, and spatial smoothing. Spatial smoothing can be reduced by applying spatial filters and by using methods like EEG brain imaging. In EEG brain imaging, a model of the head conductivity is applied to reconstruct a possible and plausible EEG source distribution [33]. Although the problem is heavily underdetermined, the results show a substantially improved spatial resolution as well as interpretability [21]. With this method, high gamma band EEG activity is successfully reconstructed, albeit not on a single trial level for BCI application and, of course, already lost information cannot be reconstructed [34]-[36]. Also the effect of signal attenuation cannot be undone. Signal attenuation with constant noise level leads to a reduced signal-to-noise ratio and makes it in turn more difficult or even impossible to access the signal of interest, especially in single trial analysis. Furthermore, it is not totally clear yet how AP spiking rates, as used in invasive BCIs, are transformed into low frequency components of the EEG, as they are typically utilized in non-invasive BCIs.

Concluding our view on the controversy, invasive BCIs measure LFP signals directly, whereas non-invasive BCIs measure modified LFP signals. The modifications are not fully understood, cannot be undone yet and are the reason for the poorer performance of non-invasive BCIs. But although invasive and non-invasive BCI signals look quite different, the origin of electrical BCIs signals is the same.

\section{Acknowledgements}

This work has been supported by the Horizon2020 EU research project "MoreGrasp” (H2020-ICT-2014-1 643955) and by the ERC Consolidator Grant 681231 "Feel Your Reach".

\section{References}

[1] Wolpaw, J.R., Birbaumer, N., McFarland, D.J., Pfurtscheller, G. and Vaughan T.M. (2002) Brain-Computer Interfaces for Communication and Control. Clinocal Neurophysiology, 113, 767-791. http://dx.doi.org/10.1016/S1388-2457(02)00057-3

[2] Millán, J.d.R., Rupp, R., Müller-Putz, G.R., Murray-Smith, R., Giugliemma, C., Tangermann, M., Vidaurre, C., Cincotti, F., Kübler, A., Leeb, R., Neuper, C., Müller, K.-R. and Mattia, D. (2010) Combining Brain-Computer Interfaces and Assistive Technologies: State-of-the-Art and Challenges. Frontiers in Neuroscience, 4, 161. http://dx.doi.org/10.3389/fnins.2010.00161

[3] Kübler, A., Nijboer, F., Mellinger, J., Vaughan, T.M., Pawelzik, H., Schalk, G., McFarland, D.J., Birbaumer, N. and Wolpaw, J.R. (2005) Patients with ALS Can Use Sensorimotor Rhythms to Operate a Brain-Computer Interface. Neurology, 64, 1775-1777. http://dx.doi.org/10.1212/01.WNL.0000158616.43002.6D

[4] Rupp, R., Rohm, M., Schneiders, M., Kreilinger, A. and Müller-Putz, G.R. (2015) Functional Rehabilitation of the Paralyzed Upper Extremity After Spinal Cord Injury by Noninvasive Hybrid Neuroprostheses. Proceedings of the IEEE, 103, 954-968. http://dx.doi.org/10.1109/JPROC.2015.2395253 
[5] Müller-Putz, G.R., Brunner, C., Bauernfeind, G., Blefari, M.L., del R. Millan, J., Real, R., Kübler, A., Mattia, D., Ramsey, N., Blankertz, B., Reuderink, B., Birbaumer, N., Salomon, P., van Steensel, M., Soekader, S., Pichiorri, F., de Pobes, A., Schettini, F., Höhne, J., Miralles, F., Otal, B., Guger, C., Ortner, R., Poel, M., Nijholt, A. and Opisso, E. (2015) The Future of Brain/Neural Computer Interaction: Horizon 2020. http://dx.doi.org/10.3217/978-3-85125-379-5

[6] Zander, T.O. and Kothe, C. (2011) Towards Passive Brain-Computer Interfaces: Applying Brain-Computer Interface Technology to Human-Machine Systems in General. Journal of Neural Engineering, 8, 025005. http://dx.doi.org/10.1088/1741-2560/8/2/025005

[7] Carmena, J.M., Lebedev, M.A., Crist, R.E., O’Doherty, J.E., Santucci, D.M., Dimitrov, D.F., et al. (2003) Learning to Control a Brain-Machine Interface for Reaching and Grasping by Primates. PLoS Biology, 1, E42. http://dx.doi.org/10.1371/journal.pbio.0000042

[8] Hochberg, L.R., Serruya, M.D., Friehs, G.M., Mukand, J.A., Saleh, M., Caplan, A.H., Branner, A., Chen, D., Penn, R.D. and Donoghue, J.P. (2006) Neuronal Ensemble Control of Prosthetic Devices by a Human with Tetraplegia. Nature, 442, 164-171. http://dx.doi.org/10.1038/nature04970

[9] Hochberg, L.R., Bacher, D., Haddadin, S., Jarosiewicz, B., Masse, N.Y., Simeral, J.D., Vogel, J., Liu, J., Cash, S.S., van der Smagt, P. and Donoghue, J.P. (2012) Reach and Grasp by People with Tetraplegia Using a Neurally Controlled Robotic Arm. Nature, 485, 372-375. http://dx.doi.org/10.1038/nature11076

[10] Wodlinger, B., Schwartz, A.B., Downey, J.E., Boninger, M.L., Collinger, J.L. and Tyler-Kabara, E.C. (2015) TenDimensional Anthropomorphic Arm Control in a Human Brain-Machine Interface: Difficulties, Solutions, and Limitations. Journal of Neural Engineering, 12, 016011. http://dx.doi.org/10.1088/1741-2560/12/1/016011

[11] Blabe, C.H., Gilja, V., Chestek, C.A., Shenoy, K.V., Anderson, K.D. and Henderson, J.M. (2015) Assessment of Brain-Machine Interfaces from the Perspective of People with Paralysis. Journal of Neural Engineering, 12, 043002. http://dx.doi.org/10.1088/1741-2560/12/4/043002

[12] Collinger, J.L., Boninger, M.L., Bruns, T.M., Curley, K., Wang, W. and Weber, D.J. (2013) Functional Priorities, Assistive Technology, and Brain-Computer Interfaces after Spinal Cord Injury. Journal of Rehabilitation Research \& Development, 50, 145-160. http://dx.doi.org/10.1682/JRRD.2011.11.0213

[13] Müller-Putz, G.R., del R. Millan, J., Schalk, G. and Müller, K.R. (2015) The Plurality of Human Brain-Computer Interfacing [Scanning the Issue]. Proceedings of the IEEE, 103, 868-870. http://dx.doi.org/10.1109/JPROC.2015.2425835

[14] David, O., Kilner, J.M. and Friston K.J. (2006) Mechanisms of Evoked and Induced Responses in MEG/EEG. NeuroImage, 31, 1580-1591. http://dx.doi.org/10.1016/j.neuroimage.2006.02.034

[15] Botrel, L., Holz, E.M. and Kübler, A. (2015) Brain Painting V2: Evaluation of P300-Based Brain-Computer Interface for Creative Expression by an End-User Following the User-Centered Design. Brain-Computer Interfaces, 2, 135-149. http://dx.doi.org/10.1080/2326263X.2015.1100038

[16] Holz, E.M., Botrel, L., Kaufmann, T. and Kübler, A. (2015) Long-Term Independent Brain-Computer Interface Home Use Improves Quality of Life of a Patient in the Locked-In State: A Case Study. Archives of physical medicine and rehabilitation, 96, 16-26. http://dx.doi.org/10.1016/j.apmr.2014.03.035

[17] Holz, E.M., Botrel, L. and Kübler, A. (2015) Independent Home Use of Brain Painting Improves Quality of Life of Two Artists in the Locked-In State Diagnosed with Amyotrophic Lateral Sclerosis. Brain-Computer Interfaces, 2, 117-134. http://dx.doi.org/10.1080/2326263X.2015.1100048

[18] Kaufmann, T., Völker, S., Gunesch, L. and Kübler, A., (2012) Spelling Is Just a Click Away-A User-Centered Brain-Computer Interface Including Auto-Calibration and Predictive Text Entry. Frontiers in Neuroscience, 6, 72. http://dx.doi.org/10.3389/fnins.2012.00072

[19] Pfurtscheller, G. and Neuper, C. (2001) Motor Imagery and Direct Brain-Computer Communication. Proceedings of the IEEE, 89, 1123-1134. http://dx.doi.org/10.1109/5.939829

[20] Leeb, R., Perdikis, S., Tonin, L., Biasiucci, A., Tavella, M., Creatura, M., Molina, A., Al-Khodairy, A., Carlson, T. and Millán, J.D., (2013) Transferring Brain-Computer Interfaces beyond the Laboratory: Successful Application Control for Motor-Disabled Users. Artificial Intelligence in Medicine, 59, 121-132. http://dx.doi.org/10.1016/j.artmed.2013.08.004

[21] Buzsáki, G., Anastassiou, C.A. and Koch C. (2012) The Origin of Extracellular Fields and Currents-EEG, ECoG, LFP and Spikes. Nature Reviews Neuroscience, 13, 407-420. http://dx.doi.org/10.1038/nrn3241

[22] Einevoll, G.T., Kayser, C., Logothetis, N.K. and Panzeri, S. (2013) Modelling and Analysis of Local Field Potentials for Studying the Function of Cortical Circuits. Nature Reviews Neuroscience, 14, 770-785. http://dx.doi.org/10.1038/nrn3599

[23] Nunez, P.L. and Srinivasan, R. (2005) Electric Fields in the Brain. The Neurophysics of EEG, Oxford University Press, Oxford. 
[24] Grimnes. S. and Martinsen, O.G. (2014) Bioimpedance and Bioelectricity Basics. 3rd Edition, Elsevier, Amsterdam.

[25] Bédard, C., Kröger, H. and Destexhe, A. (2004) Modeling Extracellular Field Potentials and the Frequency-Filtering Properties of Extracellular Space. Biophysical Journal, 86, 1829-1842. http://dx.doi.org/10.1016/S0006-3495(04)74250-2

[26] Logothetis, N.K., Kayser, C. and Oeltermann, A. (2007) In Vivo Measurement of Cortical Impedance Spectrum in Monkeys: Implications for Signal Propagation. Neuron, 55, 809-823. http://dx.doi.org/10.1016/j.neuron.2007.07.027

[27] Lindén, H., Tetzlaff, T., Potjans T.C., Pettersen, K.H., Grün, S., Diesmann, M. and Einevoll, G.T. (2001) Modeling the Spatial Reach of the LFP. Neuron, 72, 859-872. http://dx.doi.org/10.1016/j.neuron.2011.11.006

[28] Kandel, E.R., Schwartz, J.H. and Jessell, T.M. (2000) Principles of Neural Science. Fourth Edition. McGraw-Hill, New York, 324.

[29] Evarts, E.V. (1966) Pyramidal Tract Activity associated with a Conditioned Hand Movement in the Monkey. Journal of Neurophysiology, 29, 1011-1027.

[30] Schwartz, A.B., Kettner, R.E. and Georgopoulos, A.P. (1988) Primate Motor Cortex and Free Arm Movements to Visual Targets in Three-Dimensional Space. I. Relations between Single Cell Discharge and Direction of Movement. The Journal of Neuroscience, 8, 2913-2927.

[31] Hodgkin, A.L. and Huxley, A.F. (1952) A Quantitative Description of Membrane Current and Its Application to Conduction and Excitation in Nerve. The Journal of physiology, 117, 500-544. http://dx.doi.org/10.1113/jphysiol.1952.sp004764

[32] Goldman, M.S. (2004) Enhancement of Information Transmission Efficiency by Synaptic Failures. Neural Computation, 16, 1137-1162. http://dx.doi.org/10.1162/089976604773717568

[33] Michel, C.M. and Murray, M.M. (2012) Towards the Utilization of EEG Asa Brain Imaging Tool. Neuroimage, 61, 371-385. http://dx.doi.org/10.1016/j.neuroimage.2011.12.039

[34] Ball, T., Demandt, E., Mutschler, I., Neitzel, E., Mehring, C., Vogt, K., Aertsen, A. and Schulze-Bonhagea, A. (2008) Movement Related Activity in the High Gamma Range of the Human EEG. Neuroimage, 41, 302-310. http://dx.doi.org/10.1016/j.neuroimage.2008.02.032

[35] Darvas, F., Scherer, R., Ojemann, J.G., Rao, R.P., Miller, K.J. and Sorensen, L.B. (2010) High Gamma Mapping Using EEG. Neuroimage, 49, 930-938. http://dx.doi.org/10.1016/j.neuroimage.2009.08.041

[36] Seeber, M., Scherer, R., Wagner, J., Solis-Escalante, T. and Müller-Putz, G.R. (2015) High and Low Gamma EEG Oscillations in Central Sensorimotor Areas Are Conversely Modulated during the Human Gait Cycle. Neuroimage, 112, 318-326. http://dx.doi.org/10.1016/j.neuroimage.2015.03.045

\title{
Abbreviations
}

\author{
AP: Action Potential \\ BCI: Brain-Computer Interface \\ EEG: Electroencephalogram \\ LFP: Local Field Potential
}




\section{Submit or recommend next manuscript to SCIRP and we will provide best service for you:}

Accepting pre-submission inquiries through Email, Facebook, Linkedin, Twitter, etc A wide selection of journals (inclusive of 9 subjects, more than 200 journals)

Providing a 24-hour high-quality service

User-friendly online submission system

Fair and swift peer-review system

Efficient typesetting and proofreading procedure

Display of the result of downloads and visits, as well as the number of cited articles

Maximum dissemination of your research work

Submit your manuscript at: http://papersubmission.scirp.org/ 